\title{
EXPERIMENTAL STUDY OF IMPINGING FLOW GENERATED BY SYNTHETIC JETS ACTUATORS
}

\author{
Michal Kotek, Václav Kopecký• \\ Abstract: The paper describes experimental research of the flow impinging on \\ heated plate. The flow was generated by synthetic jet actuators. Construction of \\ these actuators is designed to excite a fluid flow in the circulatory track.
}

\section{INTRODUCTION}

Synthetic jets actuators are used to control and generate flows with vortex characteristics. Several applications of this phenomenon were designed on Academy of Science of the Czech Republic on Institute of Thermomechanics. Effects of these mechanisms were described also in papers [1], [2] published by prof. Václav Tesar. His team prepared experimental equipment studied in this work. The transfer medium in the experimental track was water.

The geometry of outlets from synthetic jets (SJ) chamber caused the excitation of flow in the circulation track. Vortex character of this flow should increase the heat transfer. In this work the flow field of designed SJ actuators was studied. Modern laser anemometric method called Particle image velocimetry was used to describe the flows in each part of SJ actuator period.

As mentioned above, these actuators were designed to improve the cooling effect in a closed circuit with heated plate. Detailed research of heat transfer in this apparatus is planed. For temperature field study is used the Planar Laser Induced Fluorescence (PLIF). Combination of PLIF and PIV measurement leads to a complex description of all attributes of researched flow.

\section{GEOMETRY OF LABORATORY MODEL AND EXPERIMENTAL SETUP}

The laboratory model for research of curved SJ effect is shown on Fig.1. This model was built from plexi-glass to make it transparent for optical measurement. Both methods (PIV and PLIF) are based on the principle of laser sheet illumination and camera capturing of the measured area. The design of laboratory model brings sustained laser sheet into the measured volume with minimum reflection on walls.

Main tablet of the model was manufactured on CNC machine from $10 \mathrm{~mm}$ thin plexi glass.

\footnotetext{
- Ing. Michal Kotek, Technical University of Liberec, Studentská 2, Liberec, Czech Republic, email: michal.kotek@tul.cz, tel: +420 485353637

- Prof. Ing. Václav Kopecký CSc. , Technical Univerzity of Liberec, Studentská 2, Liberec, Czech Republic, email: vaclav.kopecky@tul.cz, tel: +420 485353110
} 


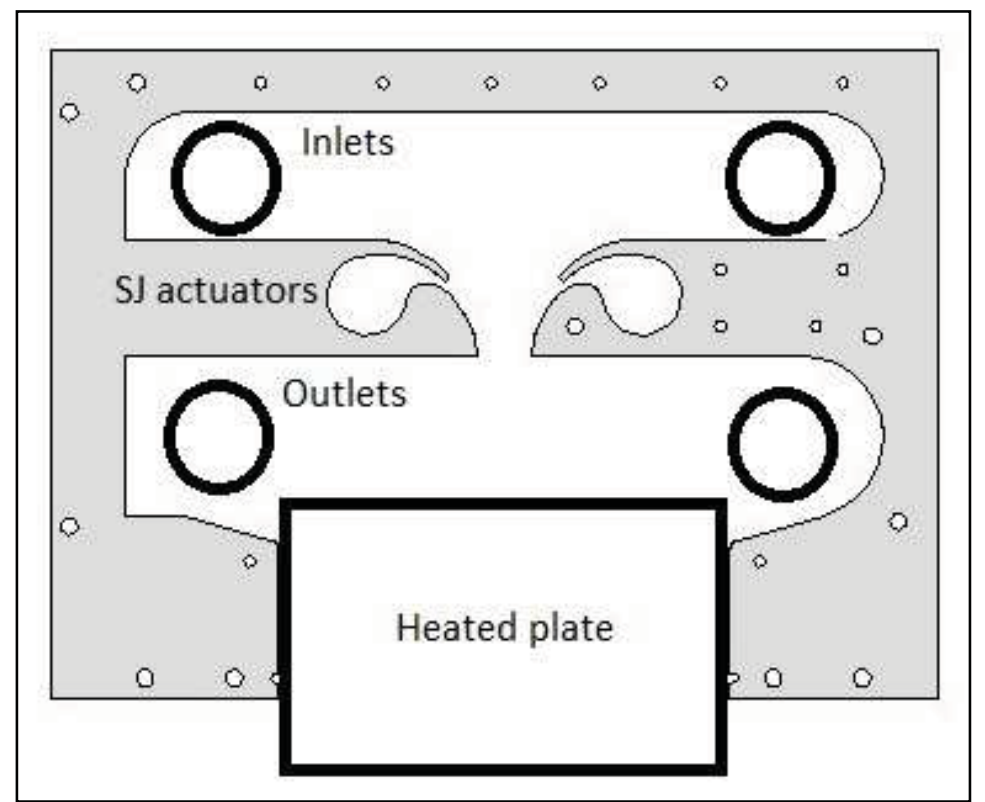

Figure $1 \ldots$ The shape of curved SJ actuators outlet, measured area with heated plate

Synthetic jets pulses were generated by a two piston mechanism connected to AC electric motor. This pulsator was driven by a frequency converter in the range of pulsing frequency $7-25 \mathrm{~Hz}$. The flow excitation ability depending on several frequencies was examined. The photo on Fig. 2 captures the pulsator mechanism and pipe junction without additional water pump.

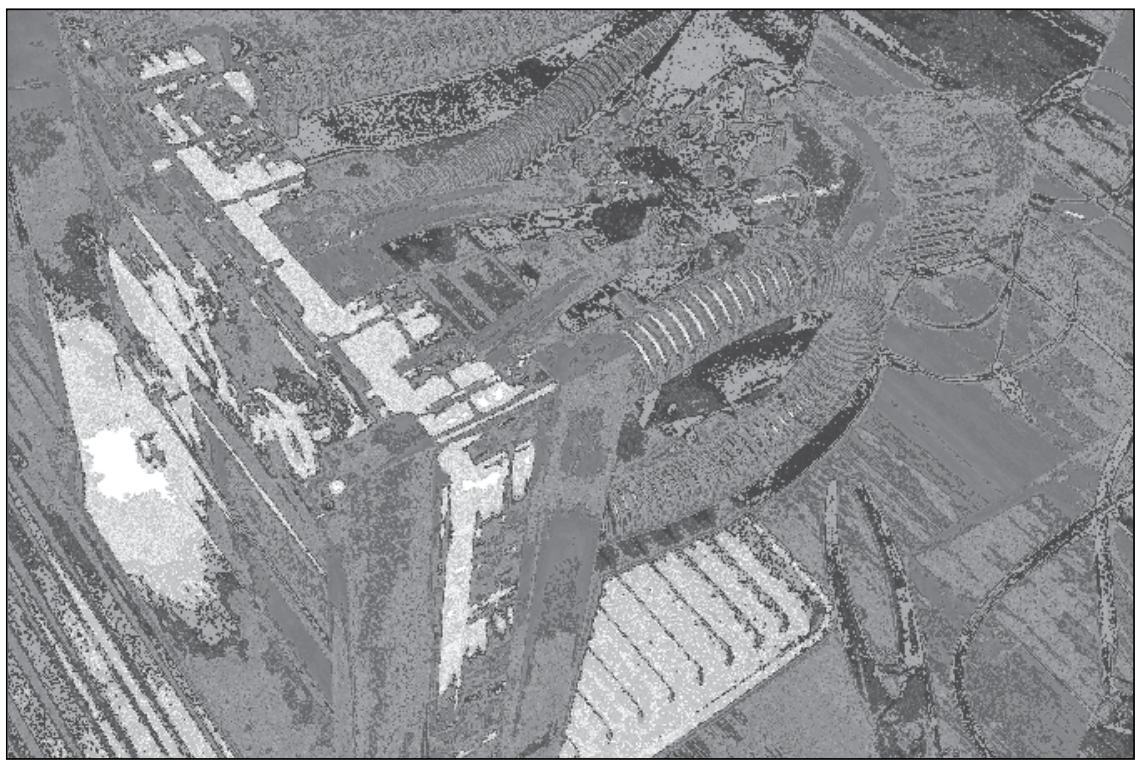

Figure 2

Laboratory model with pulsator mechanism 
Narrow measuring space and optically defective material (plexi with disturbances) produced reflections on the model walls and caused failures in the results calculation. Common procedures of image data post processing were not able to reduce all these imperfections. Routinely used polyamide seeding particles were replaced by particles coated with rhodamin layer. Rhodamin coating brought to pass the fluorescence effect instead of standard refraction. These fluorescence particles excited with laser light on wave length $532 \mathrm{~nm}$ emit light on wave length $570 \mathrm{~nm}$ - particles do not shine on green $(532 \mathrm{~nm})$ but on dark orange $(570 \mathrm{~nm})$. All the reflected and scattered light on walls etc was eliminated with a suitable lens filter. On Fig. 3 the improvement of seeding particle visibility is seen.

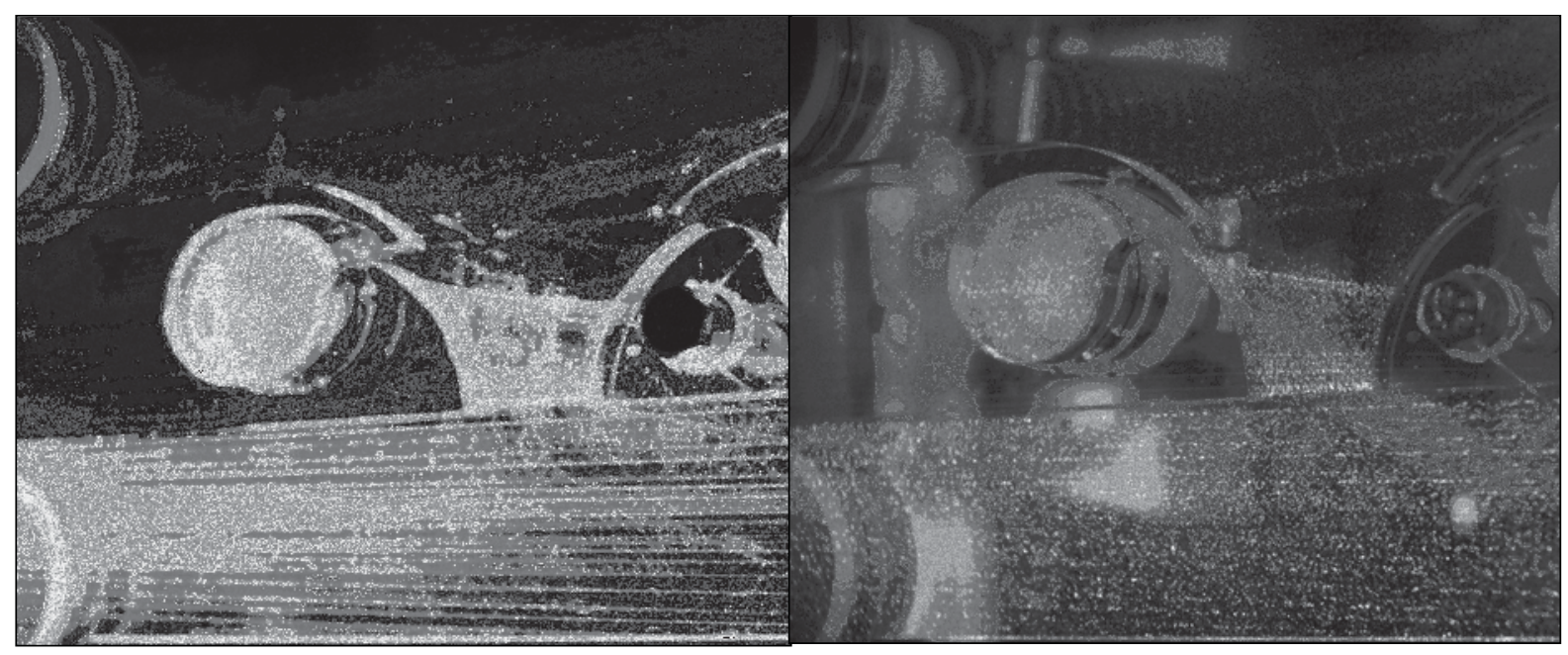

Figure $3 \ldots$ Standard seeding particles on left, fluorescence rhodamin particles on right

For flow field measurement the PIV system from Dantec Dynamics was used. The system was synchronized with the pulsator to capture the asked time step of the pulsing period. Each period moment was captured 100 times, statistic analyzing was used.

\section{RESULTS OF FLOW FIELD MEASUREMENT}

The behavior of the flow is described for two pulsator frequencies $-8 \mathrm{~Hz}$ and $16 \mathrm{~Hz}$. No additional pump was connected to the system. Results should prove the ability of suitably designed curved pulsator outlets to circulating flow in the experimental track. The series of vector maps on Fig. 4 document the flow field with $8 \mathrm{~Hz}$ pulsator frequency. 


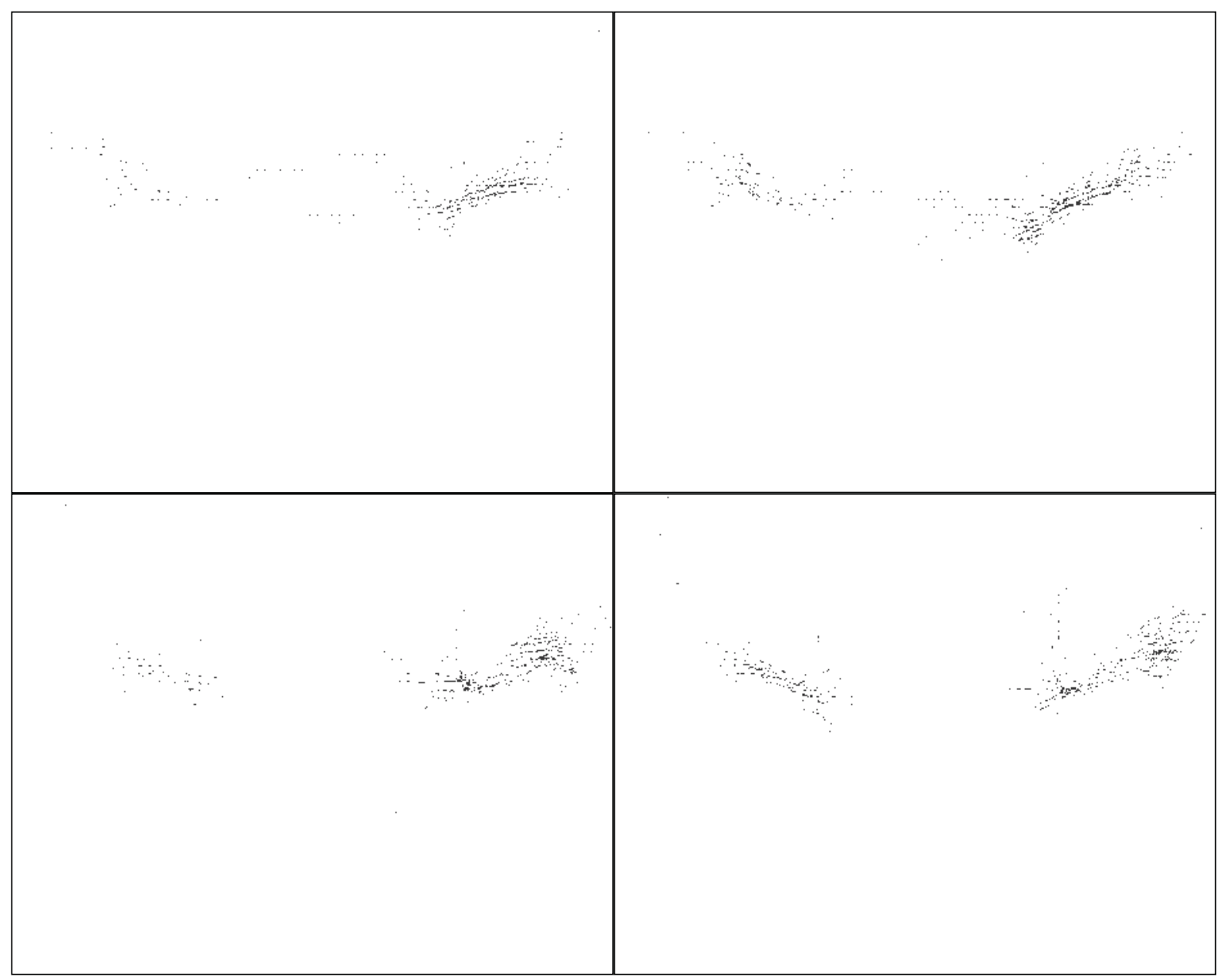

Figure $4 \ldots$ Flow fields $(8 \mathrm{~Hz})$ : left top 0T, right top $0.3 \mathrm{~T}$, left bottom $0.6 \mathrm{~T}$, right bottom $0.8 \mathrm{~T}$

Vector maps on Fig.4 clear the reason of none initiating the circulating flow. The speed of the water forced out from the pulsator chamber is low and the flow does not adhere to the curved shape, witch should lead the water to the orifice. Main volume of the water is sucked back to the opposite pulsator chamber. Final flow to the orifice has not enough energy to surpass the dissipation of the circuit.

Synthetic jets generated by the pulsators working on frequency $16 \mathrm{~Hz}$ reach higher flow speed on the chambers outlets and they ARE able to surpass the circuit dissipation. After several seconds of pulsator operating, the water starts to circulate through the circuit. Together with the vector maps also the profiles of the flow were calculated for two main areas - narrow of the curved pulsator outlet and main orifice between pulsator. 
Results of the flow are represented by vector map and streamlines. On Fig. 5 the flow in the period time $0.6 \mathrm{~T}$ is captured. Here you can see maximum outlet velocity reaching $0.12 \mathrm{~m} / \mathrm{s}$ in the outlet.

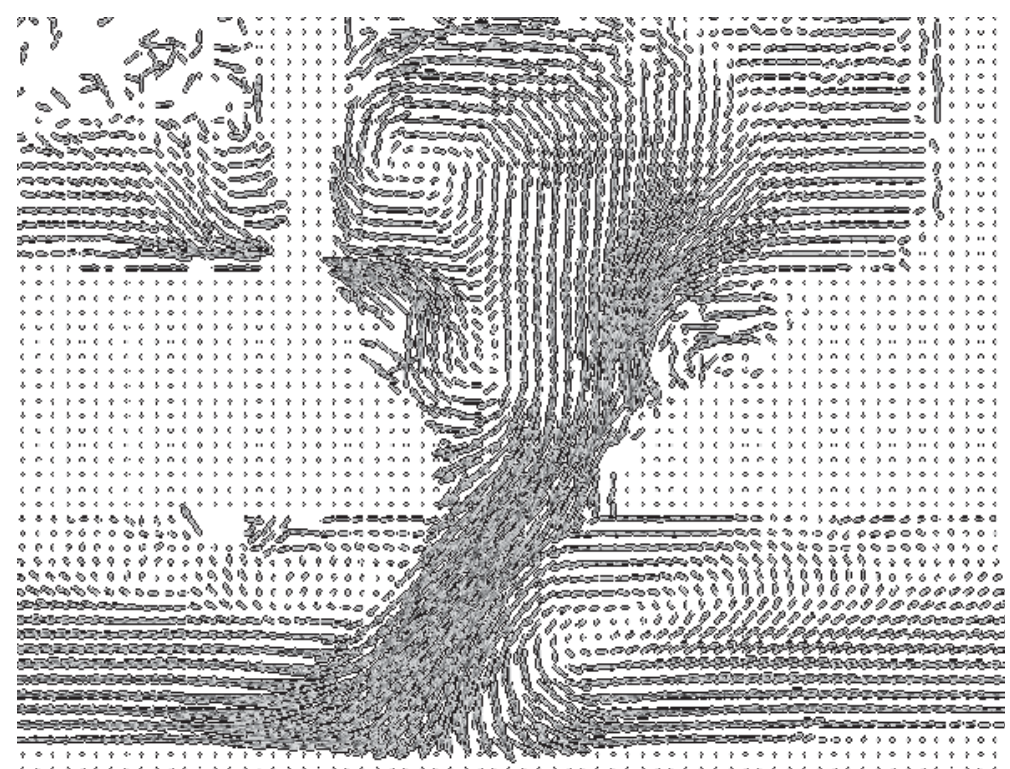

Figure 5 ... Vector map of the flow generated with the frequency $16 \mathrm{~Hz}$, time $0.6 \mathrm{~T}$

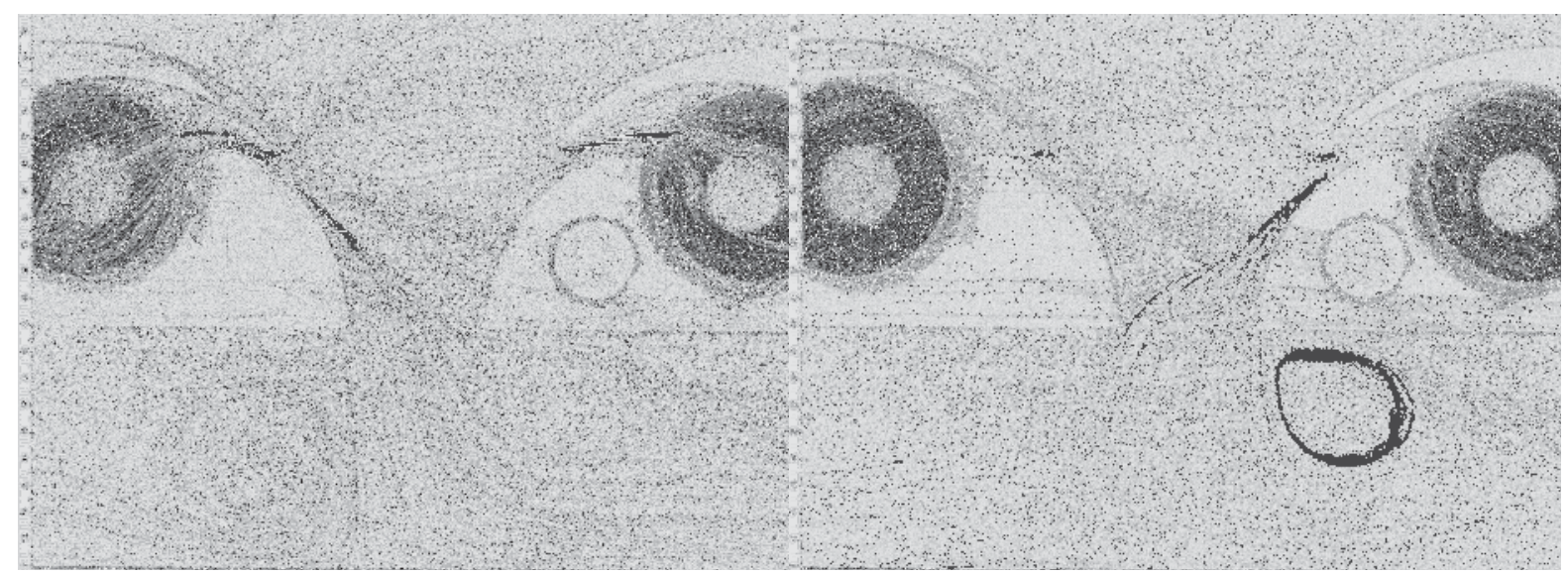

Figure 6 ... Flip-over of the flow during the period: left 0T, right 0.6T

The flip-over process in each period changes the distribution of the water into main outlets from the model. This will have dominant effect on the heat transfer.

Profile characteristics were calculated in most interesting areas of the model. Graph on Fig. 7 shows the velocity in the orifice under pulsators outlets, graph on Fig. 8 describes the velocities of the flow close to the curved wall behind the pulsator outlet. Graph shows profiles in the tangential distances from the wall in the first half of the period. 
As you can see from the flow fields above, with $16 \mathrm{~Hz}$ pulses the circulation flow raises. The speed in the orifice between pulsators reached $0.025 \mathrm{~m} / \mathrm{s}$. This value leads to a flow rate $0.31 / \mathrm{min}$ initialized in the circulation track only by the SJ actuators.

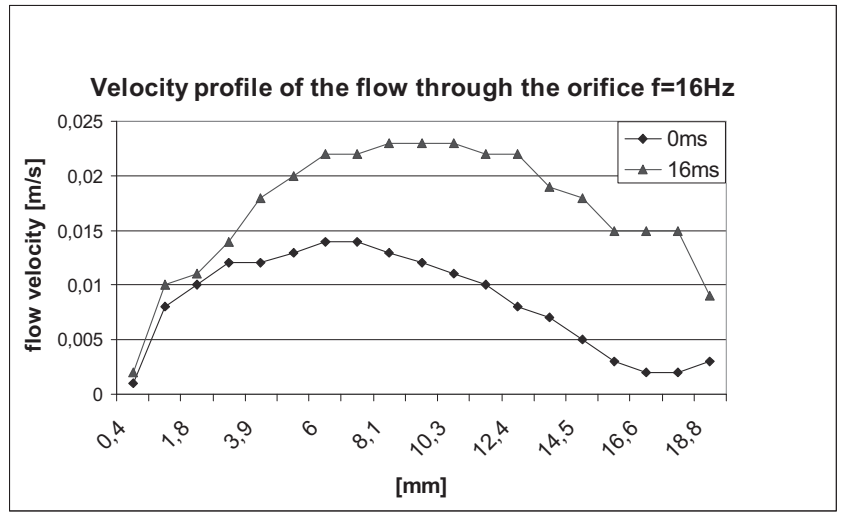

Fig.7 ... Velocity profile in the orifice

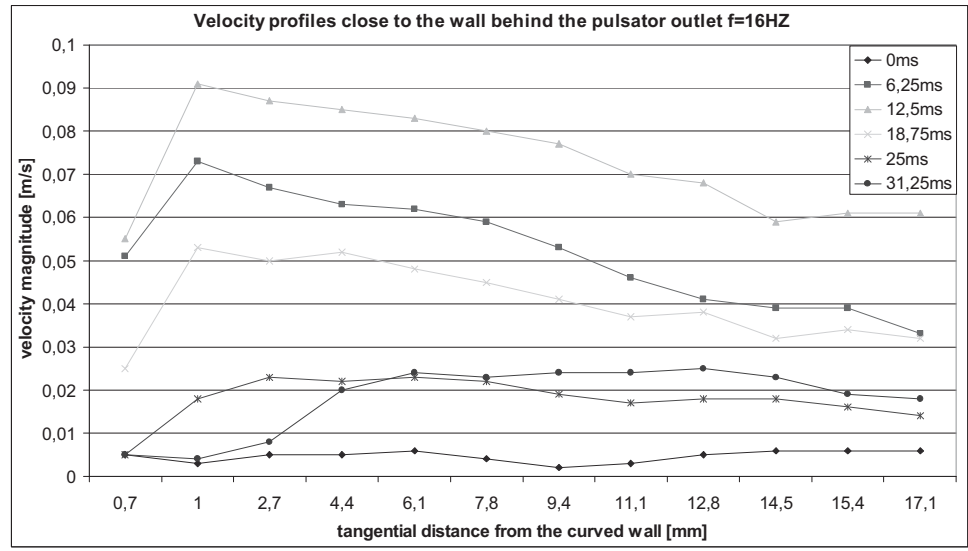

Figure 8 ...Velocity profiles close to the curved wall

\section{CONCLUSION}

Flow field in the model with SJ actuators were described. Measured results will be used for a numerical model fitting. In the present time the PLIF measurement of the temperature field are in progress. Cooperation of the flow field and temperature field results will lead to a better understanding of this phenomenon used for the heat transfer.

\section{REFERENCES}

[1] Tesař V., Trávníček Z.: „Excitational Metamorphosis of Surface Flowfield Under an Impinging Annular Jet ", Chemical Engineering Journal, pp. 312 - 316, Vol. 144(2008), Issue 2, October 2008

[2] Tesař V.: "Enhancing Impinging-Jet Heat or Mass Transfer by Fluidically Generated Flow Pulsation", Chemical Engineering Research and Design, Vol.: 87 (2009), Issue 2 (February 2009)

This work has been supported by the GACR - project num. GA101/07/1499. 